\title{
Prospective longitudinal study of frailty transitions in a community-dwelling cohort of older adults with cognitive impairment
}

\author{
Mei Sian Chong ${ }^{1,2^{*}}$, Laura Tay ${ }^{1,2}$, Mark Chan ${ }^{1,2}$, Wee Shiong Lim, ${ }^{1,2}$, Ruijing Ye ${ }^{1}$, Eng King Tan ${ }^{3,4}$ \\ and Yew Yoong Ding ${ }^{1,2}$
}

\begin{abstract}
Background: Frailty and cognitive impairment are seemingly distinct syndromes, but have a shared vulnerability to stress in older adults, resulting in poorer outcomes. Although there has been recent interest in cognitive frailty, frailty transitions in relation to cognitive deterioration in older adults with cognitive impairment have not yet been well studied. We thus aim to study frailty transitions and change in cognitive status over 1-year follow-up among subjects with cognitive impairment attending a tertiary Memory Clinic.

Methods: This is a prospective cohort study of mild cognitive impairment (MCl) and mild-moderate Alzheimer's disease (AD) community-dwelling subjects. We obtained data on clinical measures, muscle mass and physical performance measures. Cognitive status was measured using Chinese Mini-Mental State Examination (CMMSE) and Clinical Dementia Rating-Sum of Boxes (CDR-SB) scores. We measured gait speed, hand grip strength, exhaustion and weight loss at baseline, 6 and 12 months to classify subjects according to the modified Fried criteria (involving strength, gait speed, body composition and fatigue) into non-frail ( $<2$ frail categories) and frail categories ( $\geq 2$ frail categories). Frailty transitions between baseline and 12-months were assessed. We performed random effects statistical modelling to ascertain baseline predictors of longitudinal frailty scores for all subjects and within $\mathrm{MCl}$ subgroup.
\end{abstract}

Results: Among 122 subjects comprising $41 \mathrm{MCl}, 67$ mild and 14 moderate AD, 43.9, 35.8 and $57.1 \%$ were frail at baseline respectively. Frailty status regressed in $32.0 \%$, remained unchanged in $36.0 \%$, and progressed in $32.0 \%$ at 12 months. Random effects modelling on whole group showed longitudinal CDR-SB scores (coeff 0.09, $95 \%$ confidence interval (Cl) $0.03-0.15$ ) and age (coeff $0.04,95 \% \mathrm{Cl} 0.02-0.07$ ) to be significantly associated with longitudinal frailty score. Among MCl subjects, only female gender (coeff $1.28,95 \% \mathrm{Cl} 0.21-2.36$ ) was associated with longitudinal frailty score, while mild-moderate AD subjects showed similar results as those of the whole group.

Conclusions: This is the first study to show longitudinal frailty state transitions in cognitively-impaired older adults. Frailty transitions appear to be independent of progression in cognitive status in earliest stages of cognitive impairment, while mild-moderate AD subjects showed associations with age and cognitive deterioration. The potential for cognitive frailty as a separate therapeutic entity for future physical frailty prevention requires further research with a suitably powered study over a longer follow-up period.

Keywords: Frailty, Transitions, Cognitive impairment

\footnotetext{
* Correspondence: Mei_Sian_Chong@ttsh.com.sg

${ }^{1}$ Institute of Geriatrics and Active Ageing, Tan Tock Seng Hospital, 11 Jalan

Tan Tock Seng, S30843 Singapore, Singapore

Department of Geriatric Medicine, Tan Tock Seng Hospital, 11 Jalan Tan

Tock Seng, S308433 Singapore, Singapore

Full list of author information is available at the end of the article
} 


\section{Background}

Research efforts over the past decade have focused on defining the clinical and physiological characteristics of frailty and its relationship to adverse health outcomes [1, 2]. The relative lack of concrete data on the relationship between cognition and neurodegeneration in mediating frailty progression, has fuelled calls for more research into this area [3]. Recent research has focused on the proposed entity of "cognitive frailty" $[4,5]$ to describe a clinical condition that is characterized by simultaneous occurrence of physical frailty and cognitive impairment in the absence of overt dementia. This potentially could be a preventive or therapeutic target to prevent both cognitive and functional decline, and frailty progression.

Many cross-sectional studies demonstrated the relationship between general cognitive function and physical frailty [6], although less consistently with dementia [7]. Sub-domains of gait speed and grip strength appear to be more strongly associated with cognitive function, in contrast to the association of executive function with frailty $[6,8]$. Additionally, Alzheimer's disease (AD) pathology is associated with physical frailty [9]. Conversely, physical frailty has been associated with incident mild cognitive impairment (MCI) and Alzheimer's dementia, and greater rate of cognitive decline in older persons $[10,11]$. The surge of transition studies in recent years have mainly focused on physical frailty [12-15], with a relative paucity of data available for concomitant transitions in cognitive status. A recent cross-sectional study suggests a U-shaped relationship between frailty and cognition, characterized by initial dissociation with cognitive impairment and subsequent convergence at later stages [16].

Frailty among older persons have been shown to be a dynamic process, characterized by frequent transitions between frailty states over time $[15,17]$. Despite the interest in the area, most of the studies have looked at transition in frailty and health status using multistate modelling methods employing deficit counts $[14,18,19]$. More recent studies have highlighted age, medical factors $[12,20]$ and higher socioeconomic status to be protective [12]. Only one published study has looked at baseline cognition scores and frailty to be strongly associated with changes in cognitive status over time in the Canadian Study of Health and Aging (CSHA) study [21].

Functional deterioration constitutes the most significant contributor to informal and formal costs of dementia care [22]. Despite recent advances in understanding the pathophysiology of $\mathrm{AD}$ and numerous drug trials, effective interventions to prevent or delay functional decline in dementia remain elusive It has been suggested that cognitive frailty might represent the earliest spectrum of the clinical entity $[4,5]$. Because frailty represents a separate therapeutic target for dementia subjects, cognitive frailty may thus present a potential window of opportunity for targeted interventions to delay overt functional decline and disability. A deeper understanding of the trajectory and predictors of frailty transitions across the different stages of AD could allow further investigation into different stage-specific pathogenic mechanisms akin to the different biomarker stages in $\mathrm{AD}$ pathology [23], thus allowing for targeted interventions to delay functional decline and disability.

Thus, in this prospective study of community-dwelling older adults with different stages of cognitive impairment from MCI through to mild-moderate $\mathrm{AD}$ stages, we aim to study the relationship between frailty transitions and change in cognitive status over one year.

\section{Methods}

This is a prospective cohort study of $\mathrm{MCI}$ and mildmoderate probable AD community-dwelling subjects attending a specialist Memory Clinic, Cognition and Memory Disorders Service, Tan Tock Seng Hospital, Singapore between the period of December 2010 to December 2014. Ethics approval was obtained from the Domain Specific Review Board (DSRB) of the National Healthcare Group (NHG). Written consent was obtained from the patient or legally acceptable representative where appropriate.

\section{Study groups}

MCI was defined as: (a) global Clinical Dementia Rating (CDR) [24] score of 0.5; (b) subjective memory complaint which is corroborated by a reliable informant; (c) delayed recall $>1$ SD below age and education-adjusted means derived from an earlier normative study [25]; (d) relatively normal general cognitive function (Chinese Mini Mental State Examination (CMMSE) [26] score $\geq$ 21 and $\geq 24$ for subjects with $\leq 6$ and $>6$ years education respectively; and (e) largely intact activities of daily living; and [6] no clinical dementia.

Mild-moderate AD subjects fulfill diagnoses of probable AD using the National Institute of Neurological and Communicative Disorders and Stroke and Alzheimer's Disease and Related Disorders Association (NINCDSADRDA) criteria) [27] and had a global CDR score of $0.5-2.0$ respectively. We excluded subjects with a diagnosis of possible AD.

\section{Inclusion and exclusion criteria}

Subjects are eligible if they fulfilled the following: age $\geq$ 55 years; diagnosis of $\mathrm{MCI}$ or mild to moderate $\mathrm{AD}$; availability of a reliable informant; and community dwelling. We excluded subjects with presence of other central nervous conditions (stroke disease, Parkinson's disease, subdural hematoma, normal pressure hydrocephalus, and brain tumor), presence of systemic 
conditions that can contribute to CI (hypothyroidism, B12 deficiency, and hypercalcaemia), presence of any active neuropsychiatric conditions producing disability, and residence in a sheltered or nursing home. Grip strength exclusions included recent pain in the wrist or hand or a history of surgery on the upper extremity in the 3 months preceding assessment.

The validity of the overall cognitive evaluation process and CDR scoring has been previously established [24, 28]. Laboratory investigations excluding potentially reversible causes of dementia via blood tests and neuroimaging were done. A multidisciplinary consensus meeting was conducted to review all relevant results for accurate clinical phenotyping. Patients meeting study eligibility were then included in the study.

\section{Baseline data collection \\ Demographics, cognitive and functional assessment}

We collected data on: (a) baseline demographics (age, gender, years of education); (b) general cognitive function (CMMSE); (c) dementia severity (CDR global and sum-of-boxes (CDS-SB) score) [24]; (d) physical functioning (Barthel's basic activities of daily living (ADL) index [29] and Lawton's instrumental ADL (iADL) index [30]); and (e) behavioral symptoms using the Neuropsychiatric Inventory (NPI) [31] and the Diagnostic and Statistical Manual, 4th ed (DSM-IV) for Major Depression [32]. MCI subjects also underwent a neuropsychological assessment that evaluated amnestic (immediate, delayed and recognition verbal memory) and nonamnestic (language, attention, executive function and visuospatial function) domains [23].

\section{Vascular risk factor profile}

Data on hypertension, hyperlipidemia, diabetes mellitus, atrial fibrillation, peripheral vascular disease, smoking history, ischemic heart disease, and body mass index were collected. Multi-morbidity was calculated based on summation of the above factors (excluding BMI) (maximum score of 7).

\section{Neuroimaging}

Brain computed tomography $(\mathrm{CT})$ scan or magnetic resonance imaging (MRI) was performed. White matter lesions (WML) severity was graded using the Age-Related White Matter Changes (ARWMC) scale by a blinded rater (L.T.) [33]. Medial temporal atrophy (MTA) score (reflecting neurodegeneration) was scored on T1weighted coronal slices parallel to the brainstem axis and perpendicular to the hippocampal axis, by a consensus method where the scores range from 0 (no atrophy) to 4 (severe atrophy) [34].
Frailty and related nutritional and activity measurements A single blinded assessor (Y.R) measured the four components of grip strength, timed walk, unintentional weight loss $(>3 \mathrm{~kg})$ and fatigue to yield a modified Fried criteria [1], which is better operationalized in the clinical setting. The frailty scoring was done by a separate assessor (CMS). Grip strength was measured using the hydraulic hand dynamometer (North Coast@ Hydraulic Hand Dynamometer). Two trials of grip strength were obtained for each hand with all 4 trials averaged to yield a final strength score. Gait speed was based on the time taken to walk $15 \mathrm{ft}(4.5 \mathrm{~m})$. Published Asian cut-offs were used to define each frailty sub-item (grip strength $<26 \mathrm{~kg}$ for males and $<18 \mathrm{~kg}$ for females scored as 1; and gait speed $<0.8 \mathrm{~m} / \mathrm{s}$ scored as 1) [35]. Lastly, we modified 2 questions from the Center for Epidemiologic Studies-Depression Scale to assess fatigue. Participants answered yes/no to the following: (a) I felt that everything I did was an effort, and (b) I could not get "going". The frailty sub-item of fatigue was considered positive if either question was endorsed. A subject was classified as frail if the summated sub-item scores were $\geq 2$ and non-frail if $<2$. The rationale for the 4 categories and exclusion of physical activity was based on Buchmann data [10] for AD. Physical activity of subjects were obtained by way of Frenchay activity scale [36].

Nutrition was assessed using the Mini Nutritional Assessment (MNA) questionnaire [37].

\section{Muscle mass measurements}

Muscle mass (lean and fat mass of upper and lower extremity and percentage body fat) was measured via Dual energy X-ray absorptiometry (DXA) Hologic machine Discovery Series APEX 13.3-Model. DXA currently represents the more accessible technique for body composition assessment. It accurately provides estimates of lean, fat, and bone tissues in the entire body or in specific regions. The coefficient of variation $(\mathrm{CV})$ is $0.39 \%$. Appendicular Skeletal mass (ASM)/ht ${ }^{2}$ was calculated by the summation of muscle mass measured in the four limbs divided by square of height (in metres). The Short Physical Performance Battery (SPPB) [38] was used to measure physical performance.

\section{Vitamin D, lipid status and APOE genotyping}

We measured total 25-hydroxy vitamin D level with the $\mathrm{CV}$ at $17.8 \mathrm{ug} / \mathrm{L}$ was $7.2 \%$. Fasting total cholesterol, high-density lipoprotein (HDL) level, low-density lipoprotein (LDL) and triglyceride (TG) levels were obtained from the participants. Apolipoprotein $\mathrm{E}(\mathrm{APOE} \varepsilon 4)$ is a plasma cholesterol transport molecule which has been postulated to be associated with $\mathrm{AD}$. Importantly, APOE\&4 status may exert a modulatory effect on disease 
trajectory and clinical expression of disease. We performed APOE genotyping into APOE\&2, 3, 4 isoforms via restriction enzyme analysis using applied biosystems platform- ABI Prism 310 Genetic Analyser.

\section{6 and 12-month data collection}

Frailty and cognitive measures were collected at baseline, 6 and 12-month follow-up period.

Frailty transitions were defined based on differences in frailty scores between baseline and 12 months, with decrease ( $\geq-1$ point), unchanged ( 0 point) or increase $(\geq 1$ point) corresponding to regressed, unchanged and progressed frailty states respectively.

\section{Statistical analyses}

We performed univariate analysis to examine differences in baseline characteristics between the 3 cognitive subgroups (MCI, mild $A D$ and moderate $A D$ ), using analysis of variance (ANOVA) with Bonferroni correction for parametric continuous variables (as confirmed by normality testing) and Kruskal-Wallis test for nonparametric continuous variables. Chi-square test was performed for categorical variables among the 3 cognitive subgroups. Similarly, we performed univariate analyses to compare baseline characteristics among the three frailty transition subgroups (regressed, unchanged, and progressed).

We performed random effects modelling with longitudinal frailty score as the dependent variable. We included a-priori defined covariates-including age, gender, cognition-related, functional measures, biochemical and lifestyle factors for the frailty outcome. We subsequently repeated analyses separately for MCI subjects and mildmoderate AD subjects.

Statistical analyses was performed on STATA 13.0 statistical software and statistical significance taken to be $p<0.05$.

\section{Results}

Among 163 eligible subjects, 41 declined to participate in the study (10 MCI and 31 mild AD subjects). Thus, there were 122 subjects in our final sample, comprising $41 \mathrm{MCI}, 67$ mild AD and 14 moderate AD. The reasons given were refusal by their caregiver or legally acceptable representative and concerns regarding blood draw. There were no age or gender differences between subjects who accepted or decline participation in the study.

Of the 122 patients, the mean age was $75.4 \pm 7.2$ years and $40.6 \%$ were male (Table 1 ). They were predominantly of Chinese ethnicity with mean education of $7.1 \pm$ 5.0 years.
Comparison of demographics, vascular risk profile, cognitive and functional status between 3 cognitive subgroups

There were significant differences between the cognitive subgroups in terms of female preponderance 56.1, 34.3 and $21.4 \%$ male, $p<0.05)$ and smoking history (19.4, 13.4 and $14.2 \%, p<0.05)$ between MCI, mild and moderate $\mathrm{AD}$ subjects respectively. As expected, cognitive and functional performance (in terms of ADL and $\mathrm{iADL}$ ) were significantly different among the cognitive subgroups. Activity as measured on Frenchay Activity Index decreased with increasing cognitive impairment $(28.1 \pm$ 6.9, $21.6 \pm 6.6,13.8 \pm 6.9$ respectively), as did hand grip strength $(22.7 \pm 8.2, \quad 18.3 \pm 6.8,16.3 \pm 5.9$ respectively) and SPPB performance $(9.7 \pm 2.3,8.9 \pm 2.0), 6.5 \pm 2.4$ respectively) (all $p<0.05)$ (see Table 1$)$.

\section{Comparison between frailty state transitions and longitudinal cognitive performance (Fig. 1)}

In the overall group, $41.0 \%$ were frail at baseline, $37.6 \%$ frail at 6 months and $43.4 \%$ frail at 12 months. The prevalence of frailty in $\mathrm{MCI}$, mild and moderate $\mathrm{AD}$ cognitive subgroups to vary between the 6-month intervals (presented as proportions; 0.36 (95\% confidence interval (CI) (0.15-0.64) vs 0.35 (95\% CI 0.24-0.48) vs 0.50 (95\% CI $0.23-0.77$ ) at baseline; 0.29 (95\% CI $0.11-$ $0.58)$ vs 0.37 (95\% CI $0.25-0.49)$ vs 0.50 (95 \% CI $0.23-$ 0.77 ) at 6 months and 0.21 (95\% CI $0.07-0.51)$ vs 0.44 (95\% CI $0.32-0.57$ ) vs 0.67 (95\% CI $0.36-0.88$ ) at 12 months for $\mathrm{MCI}$, mild and moderate $\mathrm{AD}$ respectively) while CMMSE scores for MCI, mild and moderate AD follow a predictable linear pattern of cognitive decline $(25.2 \pm 3.4$ vs $18.4 \pm 4.6$ vs $13.3 \pm 5.4$ at baseline, and $25.0 \pm 2.4$ vs $17.8 \pm 5.1$ vs $11.0 \pm 6.3$ at 12 -month for $\mathrm{MCI}$, mild and moderate AD respectively) [30].

\section{Frailty status progression}

As a whole group, 39 subjects (32\%) had frailty regression, 44 (36 \%) remained unchanged while 39 (32\%) progressed during the one-year follow-up. Notably, among cognitive subgroups, the majority of MCI subjects either regressed $(31.7 \%)$ or remained unchanged (41.5\%) in frailty status, whereas mild and moderate AD tended to progress (47.8 and $50.0 \%$ respectively) although these observed differences did not reach statistical significance on chi-squared test $(p=0.20)$ (Fig. 2).

\section{Comparison of patient factors between 3 frailty transition status (Table 2)}

Baseline cognitive status on CMMSE and CDR-SB were not significantly different in the 3 frailty transition groups, nor did baseline measures of neurodegeneration and white matter lesions on MTA and ARWMC. Significant univariate differences were noted in smoking status, 
Table 1 Patient baseline demographics, vascular risk profile, cognitive and functional status among 3 cognitive subgroups (MCl, mild $A D$ and moderate $A D$ )

\begin{tabular}{|c|c|c|c|c|}
\hline & Total $(n=122)$ & $\mathrm{MCl}(n=41)$ & Mild AD $(n=67)$ & Moderate $\mathrm{AD}(n=14)$ \\
\hline \multicolumn{5}{|l|}{ Demographics } \\
\hline Age & $75.4(7.2)$ & $72.5(7.1)$ & $76.3(6.9)$ & $79.4(6.1)$ \\
\hline Gender (Male \%) & 40.6 & 56.1 & 34.3 & $21.4 *$ \\
\hline Race (Chinese \%) & 94.3 & 92.6 & 94.0 & 100 \\
\hline Education years & $7.1(5.0)$ & $9.9(4.7)$ & $5.8(4.6)$ & $4.9(4.5)$ \\
\hline CDR global & $0.9(0.5)$ & $0.5(0.1)$ & $0.9(0.3)$ & $1.82(0.5)^{* a, b, c}$ \\
\hline CDR Sum of boxes & $4.0(3.0)$ & $1.1(0.6)$ & $4.7(1.8)$ & $9.2(2.7)^{* a, b, c}$ \\
\hline CMMSE & $20.1(5.7)$ & $25.2(2.3)$ & $18.4(4.6)$ & $13.4(5.4)^{* a, b, c}$ \\
\hline \multicolumn{5}{|l|}{ Medical comorbidities } \\
\hline HТ (\%) & 65.5 & 58.5 & 68.7 & 71.4 \\
\hline DM (\%) & 29.5 & 24.4 & 35.8 & 14.3 \\
\hline Hyperlipidemia (\%) & 70.5 & 63.4 & 74.6 & 71.4 \\
\hline IHD (\%) & 21.3 & 26.8 & 17.9 & 21.4 \\
\hline Smoker/ex-heavy smoker (\%) & 15.5 & 19.4 & 13.4 & $14.2 *$ \\
\hline AF $(\%)$ & 3.3 & 2.4 & 4.5 & 0 \\
\hline PVD (\%) & 0.8 & 2.4 & 0 & 0 \\
\hline Multimorbidity score & $2.1(1.2)$ & $2.0(1.3)$ & $2.1(1.2)$ & $1.9(1.3)$ \\
\hline BMI & $22.9(3.2)$ & $23.1(2.6)$ & $22.8(3.6)$ & $22.8(2.4)$ \\
\hline ETOH ingestion (\%) & 12.2 & 18.2 & 10.4 & 7.1 \\
\hline Past psy problem (\%) & 4.9 & 9.8 & 1.5 & 7.1 \\
\hline MDD (\%) & 2.5 & 4.9 & 1.5 & 0 \\
\hline \multicolumn{5}{|c|}{ Neurodegeneration and wm scores } \\
\hline \multicolumn{5}{|l|}{ APOE status } \\
\hline E2/e2 & 0.8 & 0 & 1.5 & 0 \\
\hline E2/e3 & 12.3 & 17.1 & 7.5 & 21.4 \\
\hline E2/e4 & 1.6 & 2.4 & 1.5 & 0 \\
\hline E3/e3 & 47.5 & 46.3 & 52.2 & 28.6 \\
\hline E3/e4 & 32.8 & 29.3 & 31.3 & 50.0 \\
\hline E4/e4 & 4.9 & 4.9 & 6.0 & 0 \\
\hline MTA score & $1.2(1.0)(n=106)$ & $0.6(0.8)(n=38)$ & $1.5(1.0)(n=59)$ & $1.6(1.0)(n=9)$ \\
\hline ARWMC score & $4.9(4.2)(n=117)$ & $4.2(3.3)(n=38)$ & $5.0(4.8)(n=65)$ & $6.4(3.6)(n=14)$ \\
\hline \multicolumn{5}{|c|}{ Function, behaviour and burden scores } \\
\hline ADL & $97.8(5.7)$ & $99.1(1.9)$ & $97.8(5.5)$ & $93.9(11.1)^{* a, b}$ \\
\hline ¡ADL & $15.7(5.4)$ & $19.7(3.7)$ & $14.4(4.7)$ & $10.4(4.7)^{* a, b, c}$ \\
\hline NPI total severity & $2.1(1.7)$ & $1.4(1.3)$ & $2.3(1.8)$ & $2.9(1.7)^{* b, c}$ \\
\hline NPI CG distress & $3.6(5.3)$ & $1.4(2.6)$ & $4.1(5.6)$ & $7.2(6.7)^{* \mathrm{~b}, \mathrm{c}}$ \\
\hline Zarit total & $22.6(16.1)$ & $20.3(15.7)$ & $21.7(14.7)$ & $29.6(21.5)$ \\
\hline FAl total & $22.9(8.1)$ & $28.2(6.9)$ & $21.6(6.6)$ & $13.8(6.9)^{* a, b, c}$ \\
\hline
\end{tabular}


Table 1 Patient baseline demographics, vascular risk profile, cognitive and functional status among 3 cognitive subgroups (MCl, mild $A D$ and moderate $A D)$ (Continued)

Nutrition, lean mass and functional performance

\begin{tabular}{|c|c|c|c|c|}
\hline MNA total & $13.0(1.5)$ & $13.2(1.6)$ & $13.0(1.4)$ & $12.7(1.7)$ \\
\hline Grip strength & $19.5(7.5)$ & $22.7(8.2)$ & $18.3(6.8)$ & $16.3(5.9)^{* b, c}$ \\
\hline SPPB total & $8.7(2.3)$ & $9.7(2.3)$ & $8.9(2.0)$ & $6.5(2.4)^{* b, c}$ \\
\hline Lean mass measurement (ALM/ht2) & $6.6(8.2)$ & $6.2(0.9)$ & $6.9(9.8)$ & $5.5(0.7)$ \\
\hline
\end{tabular}

Abbreviations: $M C I$ mild cognitive impairment, $A D$ Alzheimer's disease, $C D R$, clinical dementia rating, CMMSE Chinese mini mental state examination (range 0-28), $H T$ hypertension, DM diabetes mellitus, IHD ischaemic heart disease, $A F$ atrial fibrillation, $P V D$ peripheral vascular disease, $B M I$, body-mass index, ETOH ethanol, $M D D$ diagnostic statistical manual definition of major depressive disorder, MTA medial temporal atrophy, ARWMC age-related white matter changes, $A D L$ activities of daily living (range $0-100$ ), CG caregiver, activities of daily living (range 0-23), NPI neuropsychiatric Inventory, FAl frenchay activity index, MNA mini-nutritional assessment, SPPB short physical performance battery, ALM/ht2 appendicular lean mass/ (height in metres) 2

${ }^{*} p<0.05$ between the 3 cognitive subgroups (ANOVA with Bonferroni correction for parametric and Kruskal-Wallis test for nonparametric continuous variables;

Chi-square test for categorical variables)

a differences between mild and moderate $A D$

${ }^{b}$ differences between $\mathrm{MCl}$ and mild $\mathrm{AD}$

'differences between $\mathrm{MCl}$ and moderate $\mathrm{AD}$

functional status (ADL and iADL), and laboratory measurements of HDL and TG (all $P<0.05$ ), whilst daily fish intake $(p=0.08)$ approached significance (Table 2$)$.

\section{Random effects modelling with longitudinal frailty score as the outcome variable}

We subsequently looked at longitudinal frailty scores via the 3 cognitive subgroups at baseline, 6 months and 12 months (Fig. 3). We performed random effects modelling with independent variables of age, gender, smoking history, functional status (iADL), lifestyle factors (fish intake) and biochemical parameters (HDL and TG levels) and longitudinal CDR-SB performance for the whole group. Age (coeff 0.09, $95 \%$ CI: 0.04-0.15, $p=0.001$ ) and cognition (CDR-SB) (coeff 0.04, $95 \%$ CI: 0.02-0.07, $p=0.000$ ) were significantly associated with frailty score (Table 3). When we performed random effects modelling only on MCI subjects $(n=41)$, only female gender was significantly associated with frailty score (coeff 1.28 , $95 \%$ CI 0.21-2.36, $p=0.019$ ) (Table 4). Subsequent analyses with mild-moderate $\mathrm{AD}$ subjects $(n=81$ showed similar findings to whole group analyses where age (coeff 0.04, $95 \%$ CI 0.02-0.07, $p=0.001$ ) and cognition (coeff $0.11,95 \%$ CI $0.05-0.17, p=0.000)$ remained significantly associated with frailty score (Table 5 ).

We performed subgroup analyses on subgroup with cognitive frailty $(n=18)$ and showed that those who progressed had more comorbidities (diabetes (4.0 compared

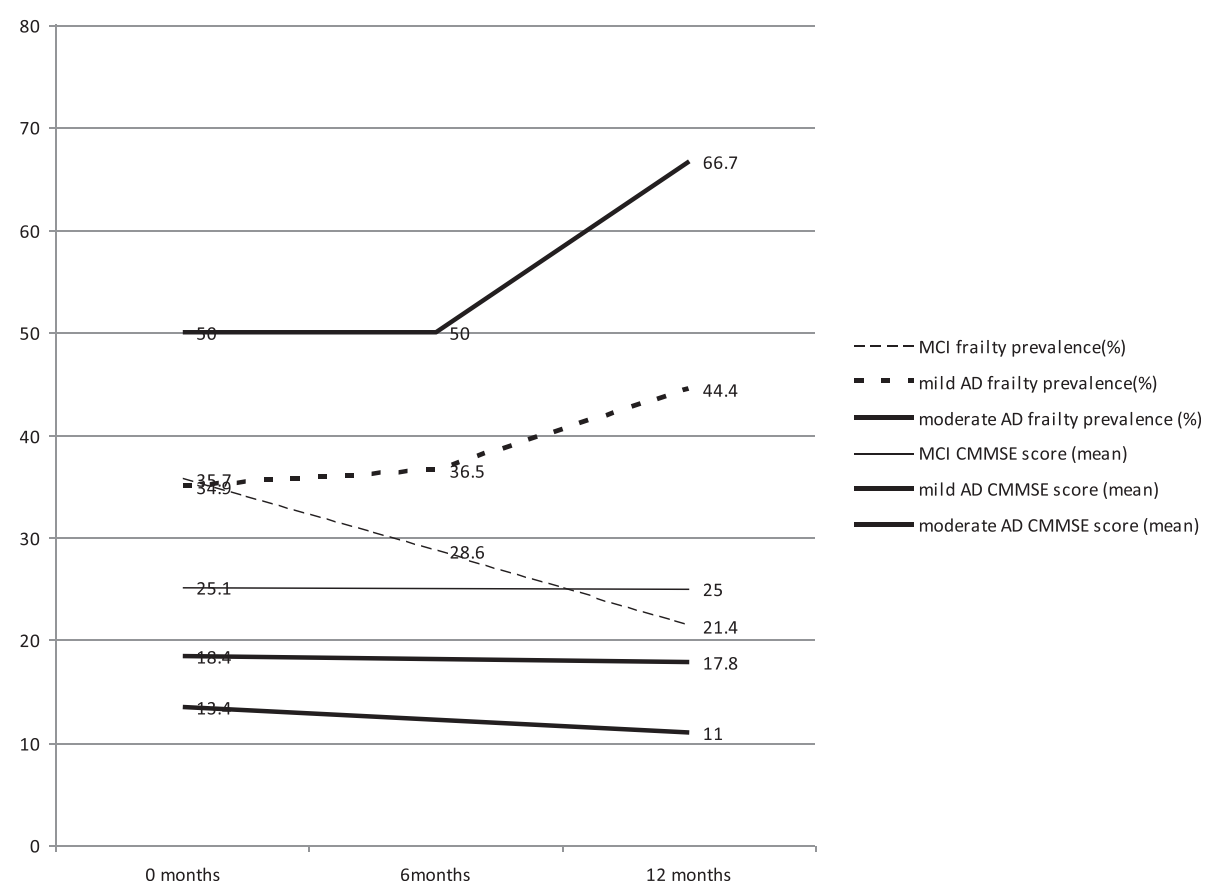

Fig. 1 Frailty prevalence at baseline, 6 months and 12 months and cognitive scores at baseline and 12 months 


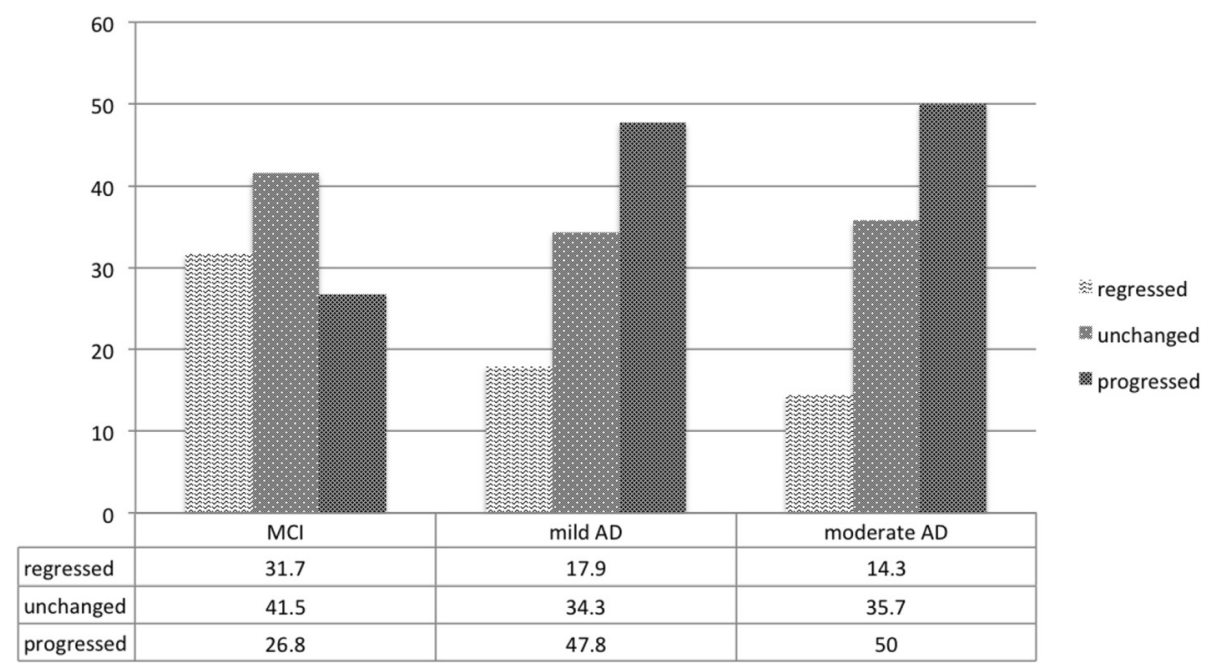

Fig. 2 Percentage of frailty transition status in the 3 cognitive subgroups ( $M C l$, mild and moderate $A D)$

to $1.7 \%$ unchanged and $0 \%$ regressed); ischaemic heart disease (33.3 compared to $66.7 \%$ unchanged and $0 \%$ regressed)), less family support (children) (40 compared to $60 \%$ regressed), were functionally more impaired (in terms of iADL) $(12.3 \pm 6.1,20.4 \pm 2.4$ unchanged and $18.7 \pm 3.7$ regressed ), had poorer performance on chair stand test $(1.0 \pm 0,4.0 \pm 0$ unchanged compared to $2.7 \pm 0.6$ regressed) with paradoxically larger lean muscle mass $(6.8 \pm 0.3 \mathrm{~kg} / \mathrm{m} 2$ progressed, $5.1 \pm 0 \mathrm{~kg} / \mathrm{m} 2$ unchanged compared to $5.3 \pm 0.4 \mathrm{~kg} / \mathrm{m} 2$ regressed) (all $p<0.05)$.

\section{Discussion}

The frailty syndrome has attracted attention as a potential therapeutic target due to its relation both as a precursor and contributor of disability in older persons. More recently, experts have focused on cognitive frailty $[4,5]$. This is the first study to demonstrate frailty state transitions over a one-year period in subjects with cognitive impairment. It is important to note that these subjects were near full independence in their basic ADLs (Table 1), indicating that they were a cohort of relatively well older adults in whom potential intervention could result in reversibility of their conditions.

Contrary to expectations of increasing frailty with increasing cognitive impairment, our previous published work showed the U-shaped relationship between frailty and increasing cognitive impairment, characterised by the initial dissociation with cognitive impairment and subsequent convergence at later stages [16]. These interesting findings have been discussed in detail in the earlier paper where these apparently contradictory differences could be partially explained, or whether a conceptual or measurement issue exists for the observed differences in frailty prevalence. These observations require a larger sample size to confirm these conclusions. Additionally, we suggested further research in the fatigue sub-item in the biological frailty model to see if the fatigue sub-item is driven by physical or psychological components [16]. Our current results demonstrate consistent results longitudinally. This is seen in the MCI stage where longitudinal frailty status and scores now show a linear decline over 12-month follow up, along with almost one-third with frailty regression, which supports the potential reversibility of cognitive frailty. These findings are further supported by random effects modelling results showing that in mild-moderate $\mathrm{AD}$ subjects, cognitive changes (as shown by CDR-SB changes) and age are directly associated with frailty progression while in MCI subjects, gender and potentially other mechanisms (unmeasured in this current study), may influence physical frailty progression. Contrary to population study findings, frailty progression in patients with established cognitive impairment appears to be associated with age and cognition, which supports the need for cognitive therapies in those already with diagnosed dementia. These initial findings also support cognitive frailty as a significant and potentially modifiable entity whereby frailty interventions may have a role in preventing physical and functional decline in cognitively impaired older adults. Our study showed differential findings to other community epidemiological studies [12] where female gender was less likely to result in decline in frailty status compared to men in MCI subjects (Table 4).

MCI remains a heterogeneous clinical construct with different underlying pathological processes (neurodegeneration, vascular, mood, metabolic) when they present in the clinical setting. In our study, we have 
Table 2 Comparison of baseline patient factors between 3 frailty transition states $(n=122)$

\begin{tabular}{|c|c|c|c|c|}
\hline & Total $(n-122)$ & Regressed $(n=39)$ & Unchanged $(n=44)$ & $\operatorname{Progressed}(n=39)$ \\
\hline \multicolumn{5}{|l|}{ Demographics } \\
\hline Age & $75.4(7.1)$ & $77.1(6.9)$ & $73.9(7.2)$ & $75.3(7.1)$ \\
\hline Gender (Male \%) & 40.1 & 38.5 & 36.4 & 46.2 \\
\hline Race (Chinese \%) & 94.3 & 92.3 & 97.7 & 92.3 \\
\hline Education years & $7.1(5.0)$ & $6.6(4.8)$ & $7.3(4.5)$ & $7.3(5.8)$ \\
\hline CDR global & $0.9(0.5)$ & $0.9(0.5)$ & $0.9(0.4)$ & $0.9(0.5)$ \\
\hline CDR Sum of boxes & $4.0(3.0)$ & $4.6(2.9)$ & $3.8(2.7)$ & $3.6(3.4)$ \\
\hline CMMSE & $20.0(6.5)$ & $19.2(5.3)$ & $20.9(5.2)$ & $20.1(6.5)$ \\
\hline \multicolumn{5}{|l|}{ Medical comorbidities } \\
\hline HT (\%) & 65.6 & 74.4 & 54.6 & 69.2 \\
\hline DM (\%) & 29.5 & 38.5 & 20.5 & 30.8 \\
\hline Hyperlipidemia (\%) & 70.5 & 66.7 & 70.5 & 74.4 \\
\hline IHD (\%) & 21.3 & 23.1 & 25.0 & 15.4 \\
\hline Smoker/ex-heavy smoker (\%) & 15.5 & 12.8 & 18.2 & $15.3 *$ \\
\hline AF (\%) & 3.3 & 5.1 & 0.0 & 5.1 \\
\hline PVD (\%) & 0.8 & 2.6 & 0 & 0 \\
\hline Multimorbidity score & $2.1(1.2)$ & $2.2(1.2)$ & $1.9(1.2)$ & $2.1(1.3)$ \\
\hline MDD (\%) & 2.5 & 2.6 & 2.3 & 2.6 \\
\hline \multicolumn{5}{|c|}{ Neurodegeneration and wm scores } \\
\hline \multicolumn{5}{|l|}{ APOE status } \\
\hline E2/e2 & 0.8 & 2.6 & 0 & 0 \\
\hline E2/e3 & 0.2 & 0.1 & 0.1 & 0 \\
\hline E2/e4 & 1.6 & 2.6 & 2.3 & 0 \\
\hline E3/e3 & 47.5 & 43.6 & 47.7 & 51.3 \\
\hline E3/e4 & 32.8 & 33.3 & 31.8 & 33.3 \\
\hline E4/e4 & 4.9 & 7.7 & 4.6 & 2.6 \\
\hline MTA score & $1.2(1.1)$ & $1.2(1.1)$ & $1.3(1.0)$ & $1.2(1.1)$ \\
\hline ARWMC score & $4.9(4.2)$ & $5.0(5.2)$ & $5.5(4.1)$ & $4.2(3.1)$ \\
\hline \multicolumn{5}{|c|}{ Function, behaviour and burden scores } \\
\hline$A D L$ & $97.8(5.8)$ & $95.9(9.0)$ & $99.0(2.8)$ & $98.5(3.1)^{* a}$ \\
\hline $\mathrm{i} A D L$ & $15.7(13.7)$ & $13.7(5.9)$ & $16.5(5.1)$ & $16.8(4.6) * a, b$ \\
\hline NPI total severity & $2.1(1.7)$ & $2.2(1.8)$ & $2.3(1.9)$ & $1.7(1.3)$ \\
\hline NPI CG distress & $3.6(5.3)$ & $3.9(6.0)$ & $4.4(5.5)$ & $2.2(3.9)$ \\
\hline Zarit total & $22.6(16.1)$ & $21.6(12.6)$ & $24.1(19.8)$ & $21.9(14.7)$ \\
\hline FAl total & $22.9(8.1)$ & $21.0(7.9)$ & $24.9(8.7)$ & $22.7(7.1)$ \\
\hline \multicolumn{5}{|c|}{ Nutrition, lean mass and functional performance } \\
\hline MNA total & $11.8(1.4)$ & $11.6(1.2)$ & $118(1.6)$ & $12.2(1.4)$ \\
\hline Grip strength & $19.5(7.5)$ & $18.1(7.3)$ & $20.6(8.0)$ & $19.8(7.1)$ \\
\hline SPPB total & $8.7(2.3)$ & $8.6(2.1)$ & $9.2(2.2)$ & $8.2(2.6)$ \\
\hline Lean mass measurement & $6.6(8.2)$ & $8.0(13.9)$ & $5.9(0.9)$ & $5.8(0.9)$ \\
\hline \multicolumn{5}{|l|}{ Treatment } \\
\hline Donepezil & 44.5 & 42.1 & 41.9 & 50.0 \\
\hline Galantamine & 0 & 0 & 0 & 0 \\
\hline Rivastigmine & 4.2 & 5.3 & 4.7 & 2.6 \\
\hline
\end{tabular}


Table 2 Comparison of baseline patient factors between 3 frailty transition states $(n=122)$ (Continued)

\begin{tabular}{|c|c|c|c|c|}
\hline Memantine & 22.7 & 34.2 & 25.6 & $7.9^{*}$ \\
\hline \multicolumn{5}{|c|}{ Socioeconomic and lifestyle factors } \\
\hline \multicolumn{5}{|l|}{ Expenses } \\
\hline More than enough & 34.4 & 60.1 & 44.4 & 63.0 \\
\hline Fair & 37.5 & 33.3 & 44.4 & 29.6 \\
\hline Not enough & 28.1 & 3.0 & 11.1 & 7.4 \\
\hline \multicolumn{5}{|l|}{ Lifestyle factors (\%) } \\
\hline \multicolumn{5}{|l|}{ Daily Activities } \\
\hline $1+h$ & 33.3 & 39.4 & 22.2 & 40.7 \\
\hline $20-59 \min$ & 31.3 & 27.3 & 38.9 & 25.9 \\
\hline$<20 \min$ & 19.8 & 18.2 & 22.2 & 18.5 \\
\hline none & 15.6 & 15.2 & 16.7 & 14.8 \\
\hline \multicolumn{5}{|c|}{ Vegetable and fruit intake (\%) } \\
\hline Daily & 83.3 & 78.8 & 86.1 & 85.2 \\
\hline$<$ Daily & 16.7 & 21.2 & 13.9 & 14.8 \\
\hline \multicolumn{5}{|l|}{ Fish intake (\%) } \\
\hline Daily & 41.7 & 30.3 & 55.6 & 37.0 \\
\hline$<$ Daily & 58.3 & 69.7 & 44.4 & $63.0(p=0.08)$ \\
\hline \multicolumn{5}{|c|}{ Social support factors (\%) } \\
\hline \multicolumn{5}{|l|}{ Neighbours } \\
\hline None & 22.9 & 27.3 & 22.2 & 18.5 \\
\hline $1-4$ & 49.0 & 45.4 & 52.8 & 48.2 \\
\hline $5-9$ & 14.6 & 12.1 & 11.1 & 22.2 \\
\hline $10+$ & 13.5 & 15.2 & 13.9 & 11.1 \\
\hline \multicolumn{5}{|c|}{ Laboratory investigations } \\
\hline Total chol & $4.9(1.1)$ & $5.1(1.1)$ & $4.8(1.2)$ & $5.0(0.9)$ \\
\hline LDL & $2.8(0.9)$ & $3.0(1.0)$ & $2.6(0.9)$ & $2.8(0.8)$ \\
\hline $\mathrm{HDL}$ & $1.6(0.7)$ & $1.6(0.5)$ & $1.6(0.7)$ & $1.7(0.7) *$ \\
\hline TG & $1.3(0.7)$ & $1.2(0.7)$ & $1.4(0.9)$ & $1.2(0.5) *$ \\
\hline Vit D level & $30.3(11.9)$ & $29.5(13.1)$ & $29.6(10.6)$ & $32.1(12.1)$ \\
\hline
\end{tabular}

Abbreviations: CDR clinical dementia rating, CMMSE Chinese mini mental state examination (range 0-28), $H T$ hypertension, $D M$ diabetes mellitus, IHD ischaemic heart disease, $A F$ atrial fibrillation, PVD peripheral vascular disease, $B M I$ body-mass index, ETOH ethanol, $M D D$ diagnostic statistical manual definition of major depressive disorder, MTA medial temporal atrophy, ARWMC age-related white matter changes, $A D L$ activities of daily living (range 0-100), CG caregiver; activities of daily living (range 0-23), NPI neuropsychiatric inventory, FAl frenchay activity index, MNA mini-nutritional assessment, SPPB short physical performance battery, $L D L$ low-density lipoprotein, $H D L$ high-density lipoprotein, $T G$ triglyceride, Vit $D$ Vitamin $D$

${ }^{*} p<0.05$ between the 3 frailty transition subgroups (ANOVA with Bonferroni correction for parametric and Kruskal-Wallis test for nonparametric continuous variables; Chi-square test for categorical variables)

${ }^{a}$ differences between frailty regressed and unchanged state

${ }^{b}$ differences between frailty regressed and progressed state

shown that $16.7 \%$ subjects with cognitive frailty (both MCI and physical frailty) had frailty progression after one year. The analyses of this small subgroup $(n=18)$ showed that those who progressed had more comorbidities (diabetes, ischaemic heart disease), less family support (children), were functionally more impaired (in terms of iADL), had poorer performance on chair stand test with paradoxically larger lean muscle mass (results not shown). This seemed independent of the
APOEe4 status or neuro-degeneration measures of medial temporal atrophy and ischaemic vascular lesions, which potentially add to further insights into the differentiation of a physically driven process, compared to a purely cognitive (neurodegeneration) driven process $[5,39,40]$. These interesting findings (despite a small sample size), requires further validation in future carefully clinically-phenotyped subjects in larger MCI studies. 


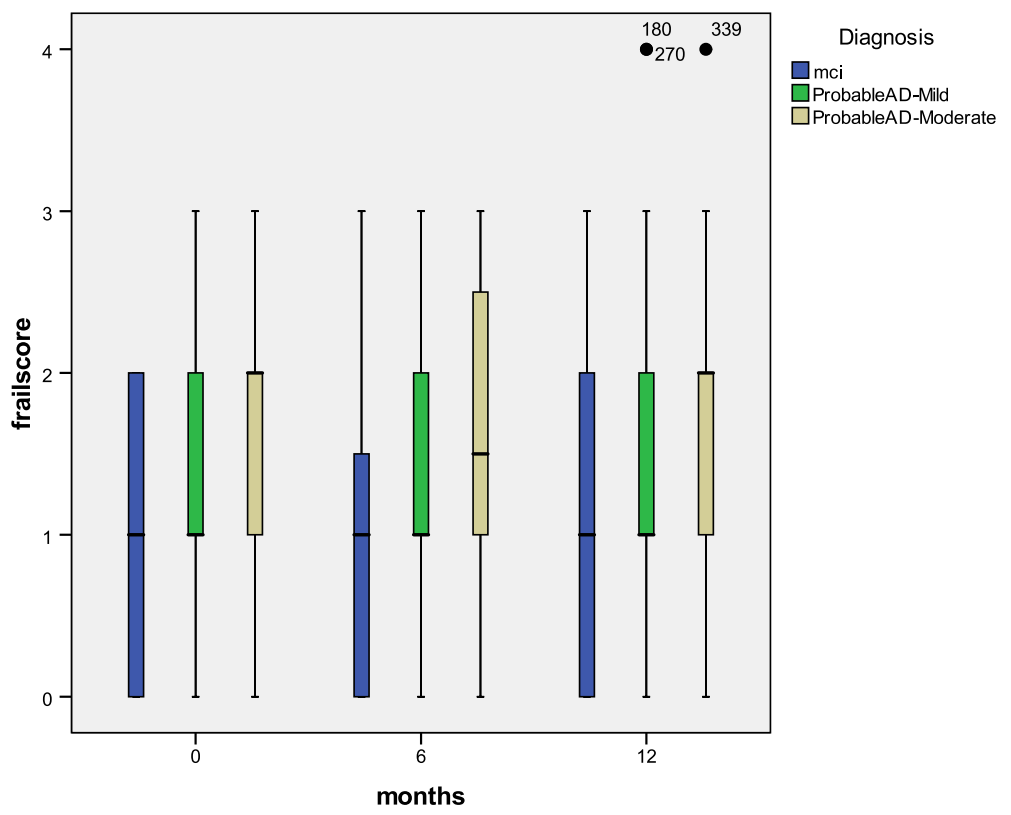

Fig. 3 Frailty score at baseline, 6 month and 12 month for 3 cognitive subgroups $(n=122)$

The strengths of our study include the comprehensive data available on both cognition and frailty measures. However, we were limited by the relatively small sample size and short follow-up period of one year. Other than the current conventional laboratory measurements, we had not included other biomarker measurements (such as inflammatory and hormonal markers) in the current paper, which might provide insights into the biological underpinnings and further understanding of the complex relationship between frailty and cognition [5]. Additionally, a matched control sample of healthy subjects without cognitive impairment could have aided in showing cognitive frailty is a separate entity, driving

Table 3 The associations of the frailty score with the various factors using the random effect model (whole group $n=122$ )

\begin{tabular}{lllll}
\hline & Coeff & Std Err & $p$-value & $95 \%$ Confidence interval \\
\hline Frailty score & & & & \\
Age & 0.04 & 0.012 & 0.000 & $0.02-0.07$ \\
Gender & 0.22 & 0.18 & 0.224 & $-0.13-0.58$ \\
Smoking & -0.01 & 0.23 & 0.964 & $-0.47-0.45$ \\
iADL & -0.004 & 0.02 & 0.80 & $-0.04-0.03$ \\
Fish intake & -0.03 & 0.15 & 0.84 & $-0.32-0.26$ \\
HDL & -0.01 & 0.13 & 0.93 & $-0.27-0.24$ \\
TG & 0.02 & 0.11 & 0.82 & $-0.19-0.24$ \\
CDR-SB & 0.09 & 0.03 & 0.001 & $0.04-0.15$
\end{tabular}

Abbreviations: Std Err standard error, iADL independent activities of daily living, $H D L$ high-density lipoprotein, $T G$ triglyceride, $C D R-S B$ clinical dementia rating sum-of-boxes score frailty progression. A separate analyses (albeit a different methodology) has suggested a concomitant proinflammatory state without concomitant endocrine deficiency, to adversely influence baseline and progressive physical frailty [41].

\section{Conclusion}

In summary, this is the first study to demonstrate longitudinal frailty state transitions in cognitively-impaired older adults. Contrary to expectations of frailty progression to be closely correlated with increasing cognitive impairment, they appear independent, especially in the earliest stage of cognitive impairment. This study lends

Table 4 The associations of the frailty score with the various factors using the random effect model ( $\mathrm{MCl}$ subjects $n=41)$

\begin{tabular}{lllll}
\hline & Coeff & Std Err & $p$-value & $95 \%$ Confidence interval \\
\hline Frailty score & & & & \\
Age & 0.04 & 0.06 & 0.49 & $-0.07-0.16$ \\
Gender & 1.28 & 0.55 & 0.02 & $0.21-2.36$ \\
Smoking & 0.07 & 0.78 & 0.94 & $-1.50-1.62$ \\
iADL & -0.10 & 0.08 & 0.22 & $-0.26-0.06$ \\
Fish intake & 0.04 & 0.53 & 0.94 & $-0.99-1.08$ \\
HDL & -0.05 & 0.30 & 0.86 & $-0.63-0.53$ \\
TG & 0.33 & 0.54 & 0.55 & $-0.74-1.39$ \\
CDR-SB & -0.31 & 0.39 & 0.43 & $-1.08-0.46$ \\
\hline
\end{tabular}

Abbreviations: Std Err standard error, iADL independent activities of daily living, $H D L$ high-density lipoprotein, $T G$ triglyceride, $C D R$-SB clinical dementia rating sum-of-boxes score 
Table 5 The associations of the frailty score with the various factors using the random effect model (mild-moderate AD subjects $n=81$ )

\begin{tabular}{lllll}
\hline & Coeff & Std Err & $p$-value & $95 \%$ Confidence interval \\
\hline Frailty score & & & & \\
Age & 0.04 & 0.01 & 0.001 & $0.02-0.07$ \\
Gender & 0.15 & 0.20 & 0.47 & $-0.25-0.54$ \\
Smoking & 0.06 & 0.27 & 0.83 & $-0.46-0.58$ \\
iADL & 0.006 & 0.02 & 0.76 & $-0.03-0.04$ \\
Fish intake & -0.11 & 0.16 & 0.49 & $-0.42-0.20$ \\
HDL & -0.15 & 0.18 & 0.42 & $-0.50-0.21$ \\
TG & 0.01 & 0.11 & 0.94 & $-0.21-0.22$ \\
CDR-SB & 0.11 & 0.03 & 0.000 & $0.05-0.17$ \\
\hline
\end{tabular}

Abbreviations: AD: Alzheimer's dementia, Std Err standard error, iADL independent activities of daily living, $H D L$ high-density lipoprotein, $T G$ triglyceride, $C D R-S B$ clinical dementia rating sum-of-boxes score

initial support to 'cognitive frailty' entity being a potentially modifiable factor for physical frailty progression. It further allowed evidence for potential refinement of this clinical construct for development of an 'at-risk' cognitive frailty phenotype. Further research is needed with a suitably powered study over a longer follow-up period with additional focus on pathogenic mechanisms to allow rapid translation of knowledge into useful therapeutic interventions.

\begin{abstract}
Abbreviations
MCl: mild cognitive impairment; AD: Alzheimer's disease; CDR-SB: clinical dementia rating- sum of boxes; DSRB: domain specific review board; NHG: National Healthcare Group; CMMSE: Chinese Mini Mental State Examination; NINCDS-ADRDA: National Institute of Neurological and Communicative Disorders and Stroke and Alzheimer's Disease and Related Disorders Association; CDR: clinical dementia rating; ADL: activities of daily living; iADL: independent activities of daily living; DSM-IV: diagnostic and statistical manual; CT: computed tomography; WML: white matter lesions; ARWMC: age-related white matter changes; MTA: medical temporal atrophy; MNA: mini nutritional assessment; DXA: dual energy $x$-ray absorptiometry; SPPB: short physical performance battery; HDL: high-density lipoprotein; LDL: low-density lipoprotein; APOE\&4: apolipoprotein E4.
\end{abstract}

\section{Competing interest}

The authors declare that they have no financial or non-financial competing interest.

\section{Author's contributions}

CMS conceived of the study, and participated in its design and coordination, performed data analyses and drafted the manuscript. LT participated in subject recruitment and helped in drafting manuscript. MC participated in subject recruitment and helped in drafting manuscript. LWS provided inputs into the study design and gave inputs in the manuscript preparation. DYY gave inputs in the manuscript preparation. YR participated in study coordination, patient recruitment and data collection. TEK carried out the gene polymorphism studies. All authors read and approved the final manuscript.

\section{Acknowledgements}

We thank Ms Ebonne Ng for her help in APOE genotyping, physiotherapist Salan Chua and occupational therapist Betty Teo in standardisation of SPPB and hand grip strength measurements. We also extend our thanks to our patients and their caregivers who have graciously consented to participation in the study. This study was funded by Lee Foundation grant 2009 and NHG
Clinician Scientist Career Scheme (CSCS)/12002. CMS is supported by NHG CSCS grant CSCS/12002 and LT is supported by NHG CSCS grant CSCS/ 13001.

\section{Author details}

${ }^{1}$ Institute of Geriatrics and Active Ageing, Tan Tock Seng Hospital, 11 Jalan Tan Tock Seng, S30843 Singapore, Singapore. ${ }^{2}$ Department of Geriatric Medicine, Tan Tock Seng Hospital, 11 Jalan Tan Tock Seng, S308433 Singapore, Singapore. ${ }^{3}$ Department of Neurology, Singapore General Hospital, Outram Rd, S169608 Singapore, Singapore. ${ }^{4}$ Duke-NUS Graduate Medical School, 8 College Road, S169857 Singapore, Singapore.

Received: 11 June 2015 Accepted: 17 December 2015

Published online: 29 December 2015

References

1. Fried LP, Tangen CM, Walston J, Newman AB, Hirsch C, Gottdiener J, et al, Frailty in older adults: evidence for a phenotype. J Gerontol A Biol Sci Med Sci. 2001;56:M146-56.

2. Gill TM, Gahbauer EA, Han L, Allore HG. Trajectories of disability in the last year of life. New Eng J Med. 2010;362:1173-80.

3. Walston J, Hadley EC, Ferrucci L, Guralnik JM, Newman AB, Studenski SA, et al. Research agenda for frailty in older adults: toward a better understanding of physiology and etiology: summary from the american geriatrics society/national institute on aging research conference on frailty in older adults. J Am Geriatr Soc. 2006:54:991-1001.

4. Canevelli M, Cesari M. Cognitive frailty: what is still missing? J Nutr Health Aging. 2015;19(3):273-5.

5. Halil M, Cemal Kizilarslanoglu M, Emin Kuyumcu M, Yesil Y, Cruz Jentoft AJ. Cognitive aspects of frailty: Mechanisms behind the link between frailty and cognitive impairment. J Nutr Health Aging. 2015;19(3):276-83.

6. Robertson DA, Savva GM, Kenny RA. Frailty and cognitive impairment- a review of the evidence and causal mechanisms. Ageing Res Reviews. 2013;12:840-51.

7. Bilotta C, Bergamaschini L, Nicolini P, Casè A, Pina G, Rossi SV, et al. Frailty syndrome diagnosed according to the study of osteoporotic fractures criteria and mortality in older outpatients suffering from Alzheimer's disease: a one-year prospective cohort study. Aging Ment Health. 2012;16:273-80.

8. Han ES, Lee Y, Kim J. Association of cognitive impairment with frailty in community-dwelling older adults. Int Psychogeriatr. 2013. doi:10.1017/ S1041610213001841.

9. Buchmann AS, Schneider JA, Leurgans S, Bennett DA. Physical frailty in older persons is associated with Alzheimer disease pathology. Neurology. 2008:71:499-504.

10. Buchman AS, Boyle PA, Wilson RS, Tang Y, Bennett DA. Frailty is associated with incident Alzheimer's disease and cognitive decline in the elderly. Psychosom Med. 2007;69(5):483-9.

11. Boyle PA, Buchman AS, Wilson RS, Leurgans SE, Bennett DA. Physical Frailty is associated with incident mild cognitive impairment in community-based older persons. J Am Geriatr Soc. 2010;58:248-55.

12. Lee JS, Auyeung TW, Leung J, Kwok T, Woo J. Transitions in frailty states among community-living older adults and their association factors. JAMDA. 2014;15:281-6.

13. Gill TM, Gahbauer EA, Han L, Allore HG. The Relationship between intervening hospitalization and transitions between frailty states. J Gerontol Med Sci. 2011;66A(11):1238-43.

14. Fallah N, Mitnitski A, Searle SD, Gahbauer EA, Gill TM, Rockwood K. Transitions in frailty status in older adults in relation to mobility: a multistate modeling approach employing a deficit count. J Am Geriatr Soc. 2011;59: 524-9.

15. Gill TM, Gahbauer EA, Allore HG, Han L. Transitions between frailty states among community-living older persons. Arch Intern Med. 2006;166:418-23.

16. Chong MS, Tay L, Chan M, Lim WS, Ye R, Wong WC, et al. Stage-specific relationship between frailty and cognitive impairment. J Frailty Aging. 2014;3(2):113-9.

17. Hardy SE, Dubin JA, Holford TR, Gill TM. Trnasitions between states of disability and independence among older persons. Am J Epid. 2004;161: 575-84.

18. Mitnitski A, Bao L, Skoog I, Rockwood K. A cross-national study of transitions in deficit counts in two birth cohorts: implications for modelling ageing. Exp Gerontol. 2007:42:241-6. 
19. Armstrong JJ, Mitnitski A, Andrew MK, Launer LJ, White LR, Rockwood K. Cumulative impact of health deficits, social vulnerabilities, and protective factors on cognitive dynamics in late life: a multistate modelling approach. Alz Res Therapy. 2015;7:38. doi:10.1186/s13195-015-0120-7.

20. Espinoza SE, Jung I, Hazuda H. Frailty transitions in the san antonio longitudinal study of aging. J Am Geriatr Soc. 2012;60:652-60.

21. Mitnitski A, Fallah N, Rockwood MR, Rockwood K. Transitions in cognitive status in relation to frailty in older adults: a Comparison of three frailty measures. J Nutr Health Aging. 2011;15:863-7.

22. Chong MS, Tan WS, Chan M, Lim WS, Ali N, Ang YY, et al. Cost of informal care for community-dwelling mild to moderate dementia patients in a developed Southeast Asian country. Int Psychogeriatr. 2013;22:1-9.

23. Jack CR Jr, Knopman DS, Jagust WJ, Petersen RC, Weiner MW, Aisen PS, et al. Tracking pathophysiological processes in Alzheimer's disease: an updated hypothetical model of dynamic biomarkers. Lancet Neurol. 2013;12:207-16.

24. Lim WS, Chin JJ, Lam CK, Lim PP, Sahadevan S. Clinical dementia rating: experience of a multi-racial Asian population. Alzheimer Dis Assoc Disord. 2005;19(3):135-42.

25. Sahadevan S, Lim JP, Tan NJ, Chan SP. Psychometric identification of early Alzheimer disease in an elderly Chinese population with differing educational levels. Alzheimer Dis Assoc Disord. 2002;16:65-72.

26. Sahadevan S, Lim PP, Tan NJ, Chan SP. Diagnostic performance of two mental status tests in the older Chinese: influence of education and age on cut-off values. Int J Geriatr Psychiatry. 2000;15(3):234-41.

27. McKhann G, Drachmann DA, Folstein M, Katzman R, Price DL, Stadlan EM. Clinical diagnosis of Alzheimer's disease - report to the NINCDS-ADRDA workgroup under the auspices of Department of Health and Human Services Task Force on Alzheimer's disease. Neurology. 1984;34:939-44.

28. Chong MS, Sahadevan S. An evidence-based clinical approach to the diagnosis of dementia. Ann Acad Med Singapore. 2003;23(6):740-9.

29. Mahoney Fl, Barthel D. Functional evaluation: the Barthel index. Md State Med J. 1965;14:56-61.

30. Barberger-Gateau P, Commenges D, Gagnon M, Letenneur L, Sauvel C, Dartigues JF. Instrumental activities of daily living as a screening tool for cognitive impairment and dementia in elderly community dwellers. J American Geriatr Soc. 1992;40:1129-34.

31. Cummings JL. The neuropsychiatric inventory: assessing psychopathology in dementia patients. Neurology. 1997;48(6):S10-6.

32. Major Depressive Episode. Diagnostic and statistical manual of mental disorders. 4th ed. Washington, DC: American Psychiatric Association; 2000. p. 349-56.

33. Wahlund LO, Barkhof F, Fazekas F, Bronge L, Augustin M, Sjögren M, et al. A new rating scale for age-related white matter changes applicable to MRI and CT. Stroke. 2001;32:1318-22.

34. Wahlund LO, Julin $\mathrm{P}$, Johansson SE, Scheltens $\mathrm{P}$. Visual rating and volumetry of the medial temporal lobe on magnetic resonance imaging in dementia: a comparative study. J Neurol Neurosurg Psychiatry. 2000;69(5):630-5.

35. Chen LK, Liu LK, Woo J, Assantachai P, Auyeung TW, Bahyah KS, et al. Sarcopenia in Asia: consensus report of the asia working group for sarcopenia. JAMDA. 2014;15(2):95-101.

36. Wade DT, Legh-Smith J, Langton HR. Social activities after stroke: measurement and natural history using the Frenchay Activities Index. Int Rehabil Med. 1985;7(4):176-81.

37. Chan M, Lim YP, Ernest A, Tan TL. Nutritional assessment in an Asian nursing home and its association with mortality. J Nutr Health Aging. 2010;14(1):23-8.

38. Guralnik JM, Simonsick EM, Ferrucci L, Glynn RJ, Berkman LF, Blazer DG, et al. A short physical performance battery assessing lower extremity function: association with self-reported disability and prediction of mortality and nursing home admission. J Gerontol Med Sci. 1994:49(2):M85-94.

39. Canevelli M, Kaleiditi E. The complex construct of mild cognitive impairment: be aware of cognitive frailty. J Frailty and Aging. 2014;3(2):87-8.

40. Canevelli M, Cesari M. Cognitive frailty: what is still missing? JNHA. 2015;19(3):273-5.

41. Tay L, Chan M, Lim WS, Chong MS. The Independent Role of Inflammation in Baseline Physical Frailty Among Older Adults with Mild Cognitive Impairment and Mild-to-Moderate Alzheimer's Disease. JNHA 2015 (in press).

\section{Submit your next manuscript to BioMed Central and we will help you at every step:}

- We accept pre-submission inquiries

- Our selector tool helps you to find the most relevant journal

- We provide round the clock customer support

- Convenient online submission

- Thorough peer review

- Inclusion in PubMed and all major indexing services

- Maximum visibility for your research

Submit your manuscript at www.biomedcentral.com/submit
( ) BioMed Central 